\title{
Prognostic Significance of Preoperative Neutrophil-to-Lymphocyte Ratio in Nonmetastatic Renal Cell Carcinoma: A Large, Multicenter Cohort Analysis
}

\author{
Seok-Soo Byun, ${ }^{1}$ Eu Chang Hwang, ${ }^{2}$ Seok Ho Kang, ${ }^{3}$ Sung-Hoo Hong, ${ }^{4}$ Jinsoo Chung, ${ }^{5}$ \\ Tae Gyun Kwon, ${ }^{6}$ Hyeon Hoe Kim, ${ }^{1}$ Cheol Kwak, ${ }^{1}$ Yong-June Kim, ${ }^{7}$ and Won Ki Lee ${ }^{8}$ \\ ${ }^{1}$ Department of Urology, College of Medicine, Seoul National University, Seoul, Republic of Korea \\ ${ }^{2}$ Department of Urology, College of Medicine, Chonnam National University, Gwangju, Republic of Korea \\ ${ }^{3}$ Department of Urology, College of Medicine, Korea University, Seoul, Republic of Korea \\ ${ }^{4}$ Department of Urology, College of Medicine, Catholic University, Seoul, Republic of Korea \\ ${ }^{5}$ Department of Urology, National Cancer Center, Goyang, Republic of Korea \\ ${ }^{6}$ Department of Urology, College of Medicine, Kyungpook National University, Daegu, Republic of Korea \\ ${ }^{7}$ Department of Urology, College of Medicine, Chungbuk National University, Cheongju, Republic of Korea \\ ${ }^{8}$ Department of Urology, College of Medicine, Hallym University, Chuncheon, Republic of Korea \\ Correspondence should be addressed to Won Ki Lee; rheewk@hanmail.net
}

Received 18 August 2016; Accepted 12 October 2016

Academic Editor: Péter Nyirády

Copyright (C) 2016 Seok-Soo Byun et al. This is an open access article distributed under the Creative Commons Attribution License, which permits unrestricted use, distribution, and reproduction in any medium, provided the original work is properly cited.

Background. The prognostic significance of the neutrophil-to-lymphocyte ratio (NLR) in nonmetastatic renal cell carcinoma (nonmRCC) is controversial, although NLR has been established as a prognostic factor in several cancers. The objective of our study was to assess the prognostic significance of preoperative NLR in non-mRCC, based on a large, multicenter cohort analysis. Methods. Totally, 1,284 non-mRCC patients undergoing surgery were enrolled from six institutions between 2000 and 2014. Recurrence-free survival (RFS) and cancer-specific survival (CSS) were calculated, and the prognostic significance of NLR was evaluated. Results. Patients with higher NLR had larger tumors $(p<0.001)$, higher T stage $(p<0.001)$, worse Eastern Cooperative Oncology Group performance status $(p<0.001)$, worse symptoms $(p=0.003)$, sarcomatoid differentiation $(p=0.004)$, and tumor necrosis $(p<0.001)$. The 5-year RFS and CSS rates were significantly lower in patients with high NLR than in those with low NLR (each $p<$ 0.001 ). Multivariate analysis identified NLR to be an independent predictor of RFS and CSS (each $p<0.05$ ). Moreover, predictive accuracy of multivariate models for RFS and CSS increased by $2.2 \%$ and $4.2 \%$, respectively, with NLR inclusion. Conclusions. Higher NLR was associated with worse clinical behavior of non-mRCC. Also, NLR was a significant prognostic factor of both RFS and CSS.

\section{Introduction}

Renal cell carcinoma (RCC) accounts for 3-4\% of all adult malignancies, and its incidence rate has been steadily increasing worldwide [1]. In the United States, the estimated numbers of new cases and deaths in 2015 were 61,560 and 14,080, respectively [1]. Therefore, it is essential to optimize decision making in treatment and prognosis of RCC and to provide better counseling for each RCC patient. Until now, many characteristics of RCC itself and patients have been suggested as possible prognostic factors. However, only a few, including pathological stage and Fuhrman grade, are undisputed prognostic factors for RCC, especially nonmetastatic RCC (nonmRCC) [2].

Inflammation has an impact on tumorigenesis and tumor progression [3]. In addition, inflammation has been recently shown to predict the prognosis of various operable cancers [4]. As inflammation is easily accessible, can be measured reliably, and can be incorporated into the tumor staging system [4], its use as a prognostic factor seems promising.

Of the many hematological and biochemical markers for systemic inflammatory response, neutrophil-to-lymphocyte 
TABLE 1: Main characteristics of recently published studies on prognostic value of neutrophil-to-lymphocyte ratio in patients with nonmetastatic renal cell carcinoma.

\begin{tabular}{|c|c|c|c|c|c|c|c|c|}
\hline \multirow{3}{*}{ Study cohort } & \multirow{3}{*}{$\begin{array}{l}\text { Study } \\
\text { cases }\end{array}$} & \multirow{3}{*}{$\begin{array}{l}\text { Histologic } \\
\text { subtype }\end{array}$} & \multirow{3}{*}{ TNM stage } & \multicolumn{5}{|c|}{ NLR } \\
\hline & & & & \multirow{2}{*}{ Value } & \multirow{2}{*}{ Cut-off } & \multicolumn{2}{|c|}{ Prognostic significance ${ }^{*}$} & \multirow{2}{*}{$\begin{array}{c}\text { Adjustment } \\
\text { variables }\end{array}$} \\
\hline & & & & & & $\mathrm{RFS}^{\#}$ & CSS & \\
\hline Lucca et al. [15] & 430 & Clear cell & T1-3 & Median 2.9 & 4.2 & Yes & NA & $\begin{array}{l}\text { Stage, grade, tumor } \\
\text { size, necrosis }\end{array}$ \\
\hline Pichler et al. [16] & 678 & Clear cell & T1-4 & Mean 3.51 & 3.3 & No & No & $\begin{array}{l}\text { Age, gender, stage, } \\
\text { grade, necrosis }\end{array}$ \\
\hline Viers et al. [17] & 827 & Clear cell & M0 & Median 3.51 & 4.0 & No & Yes & $\begin{array}{l}\text { Age, gender, } \\
\text { ECOG PS, tumor } \\
\text { size, Sx, stage, } \\
\text { grade, necrosis }\end{array}$ \\
\hline Huang et al. [18] & 218 & Papillary & $\mathrm{T} 1-3 \mathrm{Nx}$ & Median 3.1 & 3.6 & Yes & NA & $\begin{array}{l}\text { Age, gender, Sx, } \\
\text { DM, HTN, stage, } \\
\text { node, TNM group, } \\
\text { grade, necrosis, } \\
\text { ANC, ALC }\end{array}$ \\
\hline De Martino et al. [19] & 281 & $\begin{array}{l}\text { Papillary and } \\
\text { chromophobe }\end{array}$ & $\mathrm{T} 1-3 \mathrm{Nx}$ & Median 2.6 & 3.6 & Yes & NA & $\begin{array}{l}\text { Age, gender, } \\
\text { ECOG PS, stage, } \\
\text { TNM group, grade, } \\
\text { MVI, ANC, ALC }\end{array}$ \\
\hline Wen et al. [20] & 327 & All & T1-4 & Mean 2.72 & 1.7 & Yes & NA & $\begin{array}{l}\text { Age, gender, tumor } \\
\text { size, stage, subtype }\end{array}$ \\
\hline Forget et al. [21] & 227 & All & M0 & Median 3.01 & 5.0 & Yes & NA & $\begin{array}{l}\text { Age, gender, stage, } \\
\text { grade, node }\end{array}$ \\
\hline Jagdev et al. [22] & 228 & $\begin{array}{l}3 \text { major } \\
\text { subtypes }\end{array}$ & M0 & NA & NA & No & NA & NA \\
\hline Present study & 1,284 & $\begin{array}{l}3 \text { major } \\
\text { subtypes }\end{array}$ & T1-4 & Mean 2.2 & 3.7 & Yes & Yes & $\begin{array}{l}\text { Age, gender, BMI, } \\
\text { ECOG PS, Sx, } \\
\text { tumor size, stage, } \\
\text { grade, subtype, } \\
\text { sarcomatoid } \\
\text { differentiation, } \\
\text { necrosis }\end{array}$ \\
\hline
\end{tabular}

${ }^{*}$ Results from multivariate analysis.

${ }^{\#}$ RFS stands for disease-free, progression-free, and metastasis-free survival as well as recurrence-free survival.

TNM, tumor-node-metastasis; NLR, neutrophil-to-lymphocyte ratio; RFS, recurrence-free survival; CSS, cancer-specific survival; necrosis, tumor necrosis; NA, not available; ECOG PS, Eastern Cooperative Oncology Group performance status; MVI, microvascular invasion; ANC, absolute neutrophil count; ALC, absolute lymphocyte count; Sx, symptoms at presentation; DM, diabetes mellitus; HTN, hypertension.

ratio (NLR) has been introduced relatively recently [5]. Neutrophils represent the inflammatory response, whereas lymphocytes reflect cell-mediated immunity [3]. Therefore, NLR may be a better indicator of inflammation compared to existing conventional markers. Furthermore, NLR is an inexpensive, easily accessible, and widely available marker. Initially, NLR was validated as a prognostic factor of major cardiac events $[6,7]$. Since then, it has been established as a prognostic factor in several cancers including hepatocellular carcinoma and colorectal cancer [8-10].

Multiple studies suggested that NLR might be a prognostic factor in mRCC, irrespective of the treatment method [8, 11-13]. However, the few studies investigating the prognostic significance of NLR in non-mRCC have reported conflicting results [14-22]. Furthermore, previous studies were smallscale and lacked other possible prognostic factors as confounding variables (Table 1).
We assessed the prognostic significance of NLR in a large, multicenter cohort of non-mRCC patients. To our knowledge, this is the largest scale study conducted in the field, which also included the most widely accepted prognostic factors.

\section{Patients and Methods}

2.1. Patients. Approval for the study was obtained from the relevant institutional ethics committee. A total of 3,410 patients with RCC underwent curative partial or radical nephrectomy at six institutions between 2000 and 2014. We consecutively excluded 239 patients with lymph node and/or distant metastasis immediately after surgery, 574 patients who did not have any of the three major RCC subtypes (clear cell, papillary, and chromophobe variants), 351 patients with postoperative follow-up durations within 3 months, and 962 
patients with unavailable data on at least one of the relevant parameters. Only patients with complete absolute neutrophil count (ANC) and absolute lymphocyte count (ALC) data within the 2 weeks before surgery were included in the study. Finally, 1,284 non-mRCC patients (pathologically, TxN0M0) from any of the three major RCC subtypes were included in this study and retrospectively reviewed.

2.2. Variables. The characteristics of RCC and patients are detailed in Table 2.

For most patients, postoperative follow-up was scheduled every 3 months for 6 months, every 6 months for the next 3 years, and yearly thereafter. NLR was defined as the ANC divided by the ALC. The general health status was determined by the Eastern Cooperative Oncology Group performance status (ECOG PS). Tumor size was measured as the greatest diameter of the pathologic specimen. Pathologic staging was performed using the 2002 tumor-node-metastasis (TNM) classification system, and grading was performed using Fuhrman nuclear grading system. The histologic subtype was determined using the 2004 World Health Organization (WHO) international histological classification of tumors. For all specimens, urologic pathologists of each institution determined the pathologic features of the tumor. Recurrencefree survival (RFS) and cancer-specific survival (CSS) were calculated from the date of surgery to the date of recurrence and RCC-specific death, respectively, and were confirmed by imaging studies.

2.3. Statistical Analysis. The primary endpoints were RFS and CSS. The ideal cut-off level of NLR was estimated by testing all possible cut-off levels that were likely to discriminate between survival and recurrence and RCC-specific death, using the Cox proportional hazard model. The ideal cut-off level determined was then rounded to clinically relevant levels [11]. To compare the relationship between the characteristics of RCC and the patients, Student $t$-test, Pearson chi-squared test, or Fisher exact test stratified by NLR was used.

The RFS and CSS rates were calculated using the KaplanMeier method stratified by NLR, and the log-rank test was used to compare the groups. The prognostic significance of NLR as a continuous and categorical variable was evaluated using variables entered into the Cox proportional hazards model. The variables analyzed included patient age, gender, body mass index (BMI), ECOG PS, symptoms at presentation, tumor size, pathologic T stage, Fuhrman grade, histologic subtype, sarcomatoid differentiation, and tumor necrosis. The accuracy of NLR in predicting RFS and CSS was reflected by Harrell concordance index (c-index) calculated using the Cox proportional hazard models with and without the incorporation of NLR.

All tests were two-sided, and $p<0.05$ was considered statistically significant. Survival, the Cox regression method in R 3.2.2 ( $\mathrm{R}$ Development Core Team, Vienna, Austria, https://www.R-project.org/) was used to calculate the cindex, whereas IBM SPSS Statistics for Windows, version 21.0 (IBM Corp., Armonk, NY, USA) was used for other statistical assessments.

\section{Results}

3.1. The Association between Clinical and Pathologic Characteristics and NLR. A cut-off NLR level of 3.7 was estimated to be the optimal cut-off level for discriminating between patients' recurrences (hazard ratio $(\mathrm{HR})=3.049$, 95\% confidence interval $(\mathrm{CI})=2.015-4.614$, and $p<0.001)$. The same NLR cut-off level was effective for discriminating between patients' RCC-specific deaths $(\mathrm{HR}=4.947,95 \% \mathrm{CI}=2.766-$ 8.849 , and $p<0.001)$. Based on these results, the NLR cutoff level of 3.7 was used in all subsequent analyses (low NLR, $<3.7$; high NLR, $\geq 3.7$ ).

The mean follow-up period was 46.8 months for all patients (median 39 months; interquartile range, 19-69 months). The mean NLRs of patients with low and high NLR were $1.8 \pm 0.7$ and $6.0 \pm 3.2$, respectively $(p<0.001)$. Table 1 shows the association of NLR with different clinical and pathological characteristics. Patients with high NLR differed significantly from those with low NLR in various parameters. Patients with high NLR were older $(p=0.001)$ and had higher ECOG PS $(p<0.001)$ and T stage $(p<0.001)$ and larger tumors $(p<0.001)$ compared to those with low NLR. Patients with high NLR also had greater symptom ratios ( $p=$ $0.003)$, sarcomatoid differentiation ratios $(p=0.004)$, and tumor necrosis ratios $(p<0.001)$.

3.2. Recurrence-Free Survival in relation to NLR. During follow-up, 142 (11.1\%) patients had recurrence (Table 2). The 5 -year RFS rates were $71.6 \%$ in patients with high NLR and $88.2 \%$ in those with low NLR. The 5-year RFS rate was significantly lower in patients with high NLR than in those with low NLR $(p<0.001$; Figure $1(\mathrm{a}))$.

Multivariate analysis identified NLR to be an independent predictor of RFS (HR of NLR as a continuous variable $=1.081$, $p=0.028$; HR of NLR as a categorical variable $=1.788, p=$ 0.009 ; Table 3 ). The predictive accuracy of the multivariate model with NLR was $81.1 \%$, whereas that of the multivariate model without NLR was $78.9 \%$.

3.3. Cancer-Specific Survival in relation to NLR. During follow-up, 56 (4.4\%) patients died of RCC-related causes (Table 2). The 5 -year CSS rates were $84.2 \%$ in patients with high NLR and $96.4 \%$ in those with low NLR. The 5-year CSS rate was significantly lower in patients with high NLR than in those with low NLR $(p<0.001$; Figure $1(\mathrm{~b}))$.

Multivariate analysis identified NLR to be an independent predictor of CSS (HR of NLR as a continuous variable $=1.156$, $p=0.009$; HR of NLR as a categorical variable $=2.566, p=$ 0.004; Table 4). The predictive accuracy of the multivariate model with NLR was $87.9 \%$, whereas that of the multivariate model without NLR was $83.7 \%$.

\section{Discussion}

In this study, NLR was identified to be a significant prognostic factor of both RFS and CSS in patients with nonmRCC, even when the models were adjusted for other wellknown prognostic factors. The predictive accuracy of the 
TABLE 2: Association of different clinical and pathological characteristics with neutrophil-to-lymphocyte ratio in patients with nonmetastatic renal cell carcinoma.

\begin{tabular}{|c|c|c|c|c|}
\hline Variable & All & Low NLR & High NLR & $p$ value \\
\hline Number of subjects & 1,284 & 1,168 & 116 & \\
\hline $\mathrm{NLR}$, mean $\pm \mathrm{SD}$ & $2.2 \pm 1.7$ & $1.8 \pm 0.7$ & $6.0 \pm 3.2$ & $<0.001^{*}$ \\
\hline Age, mean $\pm S D$, year & $55.9 \pm 12.9$ & $55.5 \pm 12.8$ & $59.8 \pm 12.9$ & $0.001^{*}$ \\
\hline Gender & & & & $0.236^{* *}$ \\
\hline Male, $n(\%)$ & $913(71.1)$ & $825(70.6)$ & $88(75.9)$ & \\
\hline Female, $n(\%)$ & $371(28.9)$ & $343(29.4)$ & $28(24.1)$ & \\
\hline $\mathrm{BMI}$, mean $\pm \mathrm{SD}, \mathrm{kg} / \mathrm{m}^{2}$ & $24.6 \pm 3.3$ & $24.7 \pm 3.2$ & $23.8 \pm 3.4$ & $0.006^{*}$ \\
\hline ECOG PS $\geq 1, n(\%)$ & $180(14.0)$ & $148(12.7)$ & $32(27.6)$ & $<0.001^{* *}$ \\
\hline Symptoms at presentation & & & & $0.003^{* *}$ \\
\hline No symptom, $n(\%)$ & $975(75.9)$ & $900(77.1)$ & $75(64.7)$ & \\
\hline Symptom, $n(\%)$ & $309(24.1)$ & $268(22.9)$ & $41(35.3)$ & \\
\hline \multicolumn{5}{|l|}{ Tumor size } \\
\hline (1) mean $\pm \mathrm{SD}, \mathrm{cm}$ & $4.08 \pm 2.68$ & $3.94 \pm 2.54$ & $5.50 \pm 3.55$ & $<0.001^{*}$ \\
\hline (2) Category & & & & $<0.001^{* *}$ \\
\hline$<4 \mathrm{~cm}, n(\%)$ & $748(58.3)$ & $701(60.0)$ & $47(40.5)$ & \\
\hline $4-7 \mathrm{~cm}, n(\%)$ & $351(27.3)$ & $321(27.5)$ & $30(25.9)$ & \\
\hline$\geq 7 \mathrm{~cm}, n(\%)$ & $185(14.4)$ & $146(12.5)$ & $39(33.6)$ & \\
\hline Side & & & & $1.000^{* * *}$ \\
\hline Unilateral, $n(\%)$ & $1,268(98.8)$ & $1,153(98.7)$ & $115(99.1)$ & \\
\hline Bilateral, $n(\%)$ & $16(1.2)$ & $15(1.3)$ & $1(0.9)$ & \\
\hline Type of nephrectomy & & & & $<0.001^{* *}$ \\
\hline Radical, $n(\%)$ & $634(49.4)$ & $552(47.3)$ & $82(70.7)$ & \\
\hline Partial, $n(\%)$ & $650(50.6)$ & $616(52.7)$ & $34(29.3)$ & \\
\hline Method of surgery & & & & $0.042^{* *}$ \\
\hline Open, $n(\%)$ & $697(54.3)$ & $628(53.8)$ & $69(59.5)$ & \\
\hline Laparoscopic, $n(\%)$ & $316(24.6)$ & $283(24.2)$ & $33(28.4)$ & \\
\hline Robot, $n(\%)$ & $271(21.1)$ & $257(22.0)$ & $14(12.1)$ & \\
\hline T stage & & & & $<0.001^{* *}$ \\
\hline $\mathrm{T} 1, n(\%)$ & $1,016(79.1)$ & $945(80.9)$ & $71(61.2)$ & \\
\hline $\mathrm{T} 2, n(\%)$ & $89(6.9)$ & $75(6.4)$ & $14(12.1)$ & \\
\hline $\mathrm{T} 3-4, n(\%)$ & $179(13.9)$ & $148(12.7)$ & $31(26.7)$ & \\
\hline Fuhrman's grade & & & & $0.561^{* *}$ \\
\hline G1-2, $n(\%)$ & $664(51.7)$ & $607(52.0)$ & $57(49.1)$ & \\
\hline $\mathrm{G} 3-4, n(\%)$ & $620(48.3)$ & $561(48.0)$ & $59(50.9)$ & \\
\hline Histologic subtype & & & & $0.042^{* *}$ \\
\hline Clear cell, $n(\%)$ & $1,114(86.8)$ & $1,017(87.1)$ & $97(83.6)$ & \\
\hline Papillary, $n(\%)$ & $87(6.8)$ & $73(6.3)$ & $14(12.1)$ & \\
\hline Chromophobe, $n(\%)$ & $83(6.5)$ & $78(6.7)$ & $5(4.3)$ & \\
\hline Sarcomatoid differentiation, yes, $n(\%)$ & $29(2.3)$ & $22(1.9)$ & $7(6.0)$ & $0.004^{* *}$ \\
\hline Tumor necrosis, yes, $n(\%)$ & $208(16.2)$ & $174(14.9)$ & $34(29.3)$ & $<0.001^{* *}$ \\
\hline Recurrence, $n(\%)$ & $142(11.1)$ & $114(9.8)$ & $28(24.1)$ & $<0.001^{* *}$ \\
\hline RCC-specific death, $n(\%)$ & $56(4.4)$ & $40(3.4)$ & $16(13.8)$ & $<0.001^{* *}$ \\
\hline
\end{tabular}

NLR, neutrophil-to-lymphocyte ratio; low NLR, $<3.7$; high NLR, $\geq 3.7$; BMI, body mass index; ECOG PS, Eastern Cooperative Oncology Group performance status; RCC, renal cell carcinoma; $n$, number of subjects; SD, standard deviation.

${ }^{*}$ Student $t$-test.

** Pearson's chi-square test.

${ }^{* * * *}$ Fisher's exact test. 
TABLE 3: Multivariate analyses predicting probability of cancer recurrence in relation to the neutrophil-to-lymphocyte ratio in patients with nonmetastatic renal cell carcinoma.

\begin{tabular}{|c|c|c|c|c|c|c|}
\hline \multirow{2}{*}{ Variables } & \multicolumn{3}{|c|}{ NLR as a continuous variable } & \multicolumn{3}{|c|}{ NLR as a categorical variable } \\
\hline & HR & $95 \% \mathrm{CI}$ & $p$ value & HR & $95 \% \mathrm{CI}$ & $p$ value \\
\hline Age & 1.011 & $0.997-1.025$ & 0.134 & 1.011 & $0.997-1.026$ & 0.123 \\
\hline \multicolumn{7}{|l|}{ Gender } \\
\hline Female versus male & 0.873 & $0.588-1.296$ & 0.502 & 0.876 & $0.591-1.299$ & 0.510 \\
\hline BMI & 0.959 & $0.907-1.015$ & 0.146 & 0.959 & $0.907-1.014$ & 0.146 \\
\hline \multicolumn{7}{|l|}{ ECOG PS } \\
\hline$\geq 1$ versus 0 & 1.936 & $1.270-2.950$ & 0.002 & 1.900 & $1.244-2.902$ & 0.003 \\
\hline Symptoms at presentation & 1.185 & $0.811-1.731$ & 0.380 & 1.208 & $0.830-1.758$ & 0.325 \\
\hline Tumor size & 1.011 & $1.005-1.017$ & 0.001 & 1.011 & $1.004-1.017$ & 0.001 \\
\hline T stage & & & 0.009 & & & 0.010 \\
\hline $\mathrm{T} 2$ versus $\mathrm{T} 1$ & 1.384 & $0.745-2.571$ & 0.303 & 1.376 & $0.743-2.550$ & 0.310 \\
\hline $\mathrm{T} 3-4$ versus $\mathrm{T} 1$ & 2.068 & $1.281-3.340$ & 0.003 & 2.050 & $1.267-3.314$ & 0.003 \\
\hline \multicolumn{7}{|l|}{ Fuhrman's grade } \\
\hline G3-4 versus G1-2 & 1.974 & $1.352-2.882$ & $<0.001$ & 1.958 & $1.340-2.863$ & 0.001 \\
\hline Histologic subtype & & & 0.012 & & & 0.019 \\
\hline pRCC versus cRCC & 1.044 & $0.582-1.872$ & 0.886 & 1.029 & $0.575-1.841$ & 0.924 \\
\hline chRCC versus cRCC & 0.104 & $0.023-0.467$ & 0.003 & 0.132 & $0.032-0.545$ & 0.005 \\
\hline Sarcomatoid differentiation & 2.095 & $1.061-4.137$ & 0.033 & 2.004 & $1.010-3.977$ & 0.047 \\
\hline Tumor necrosis & 1.255 & $0.817-1.927$ & 0.300 & 1.265 & $0.825-1.939$ & 0.282 \\
\hline \multicolumn{7}{|l|}{ NLR } \\
\hline (1) Continuous & 1.081 & $1.009-1.160$ & 0.028 & & & \\
\hline (2) High versus low NLR & & & & 1.788 & $1.153-2.771$ & 0.009 \\
\hline
\end{tabular}

NLR, neutrophil-to-lymphocyte ratio; low NLR, <3.7; high NLR, $\geq 3.7$; BMI, body mass index; ECOG PS, Eastern Cooperative Oncology Group performance status; cRCC, clear cell renal cell carcinoma; pRCC, papillary renal cell carcinoma; chRCC, chromophobe renal cell carcinoma; HR, hazard ratio; CI, confidence interval.

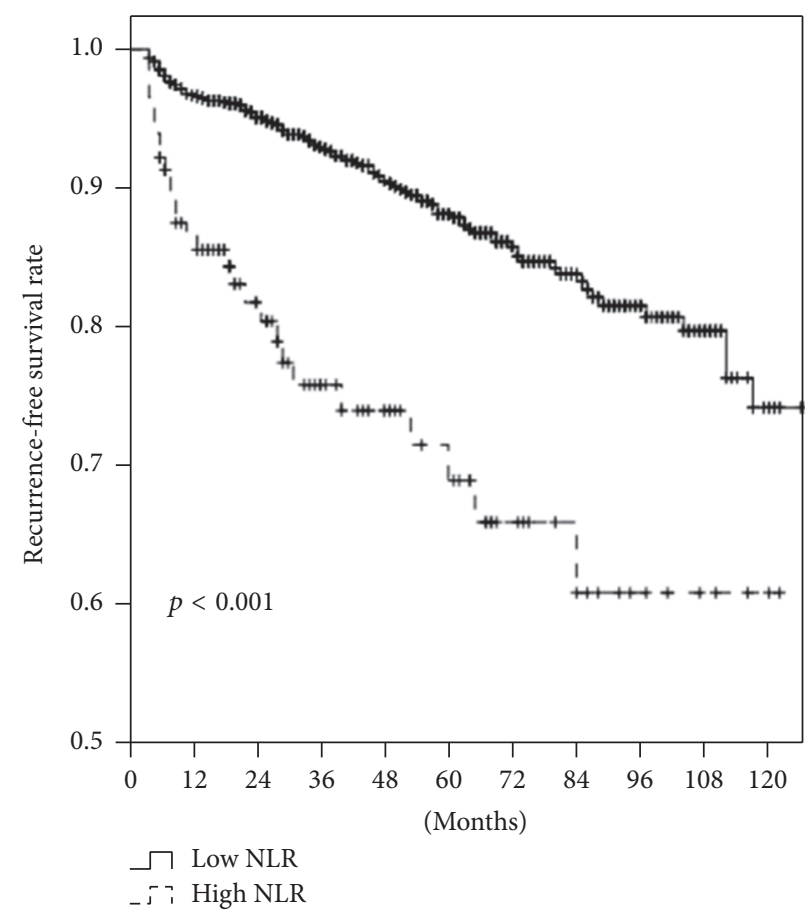

(a)

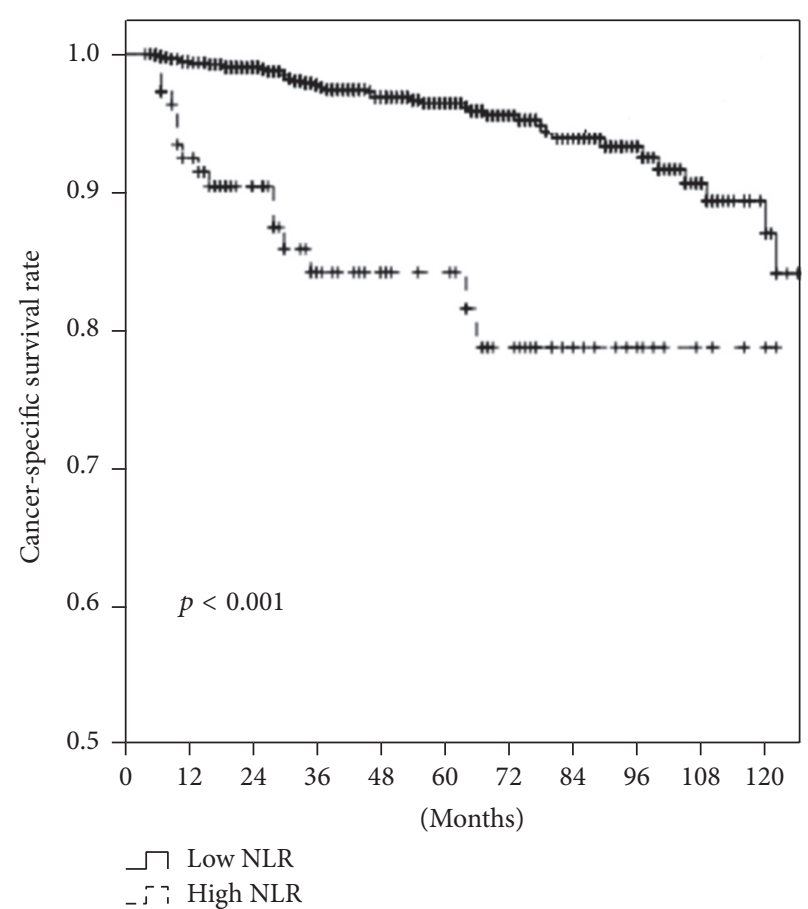

(b)

FiguRE 1: Kaplan-Meier curve for recurrence-free survival (a) and cancer-specific survival (b) for patients with nonmetastatic renal cell carcinoma according to neutrophil-to-lymphocyte ratio. NLR, neutrophil-to-lymphocyte ratio. 
TABLE 4: Multivariate analyses predicting probability of cancer-specific death in relation to the neutrophil-to-lymphocyte ratio in patients with nonmetastatic renal cell carcinoma.

\begin{tabular}{|c|c|c|c|c|c|c|}
\hline \multirow{2}{*}{ Variables } & \multicolumn{3}{|c|}{ NLR as a continuous variable } & \multicolumn{3}{|c|}{ NLR as a categorical variable } \\
\hline & $\mathrm{HR}$ & $95 \%$ CI & $p$ value & HR & $95 \% \mathrm{CI}$ & $p$ value \\
\hline Age & 1.042 & $1.016-1.069$ & 0.002 & 1.044 & $1.018-1.072$ & 0.001 \\
\hline \multicolumn{7}{|l|}{ Gender } \\
\hline Female versus male & 0.652 & $0.324-1.313$ & 0.231 & 0.648 & $0.323-1.300$ & 0.222 \\
\hline BMI & 0.916 & $0.832-1.009$ & 0.074 & 0.924 & $0.840-1.017$ & 0.105 \\
\hline \multicolumn{7}{|l|}{ ECOG PS } \\
\hline$\geq 1$ versus 0 & 2.820 & $1.498-5.309$ & 0.001 & 2.672 & $1.408-5.071$ & 0.003 \\
\hline Symptoms at presentation & 1.029 & $0.558-1.897$ & 0.927 & 1.056 & $0.577-1.932$ & 0.860 \\
\hline Tumor size & 1.012 & $1.002-1.022$ & 0.015 & 1.012 & $1.002-1.022$ & 0.018 \\
\hline T stage & & & 0.022 & & & 0.020 \\
\hline $\mathrm{T} 2$ versus $\mathrm{T} 1$ & 0.665 & $0.198-2.233$ & 0.509 & 0.662 & $0.198-2.215$ & 0.503 \\
\hline $\mathrm{T} 3-4$ versus $\mathrm{T} 1$ & 2.175 & $1.025-4.617$ & 0.043 & 2.209 & $1.041-4.688$ & 0.039 \\
\hline \multicolumn{7}{|l|}{ Fuhrman's grade } \\
\hline G3-4 versus G1-2 & 2.155 & $1.141-4.072$ & 0.018 & 2.101 & $1.110-3.977$ & 0.023 \\
\hline Histologic subtype & & & 0.854 & & & 0.860 \\
\hline pRCC versus cRCC & 1.268 & $0.551-2.919$ & 0.576 & 1.257 & $0.554-2.850$ & 0.584 \\
\hline chRCC versus cRCC & 0.001 & $<0.001-5.496$ & 0.959 & 0.001 & $<0.001-6.687$ & 0.962 \\
\hline Sarcomatoid differentiation & 3.355 & $1.230-9.148$ & 0.018 & 3.092 & $1.123-8.514$ & 0.029 \\
\hline Tumor necrosis & 1.054 & $0.509-2.181$ & 0.888 & 1.097 & $0.537-2.242$ & 0.799 \\
\hline \multicolumn{7}{|l|}{ NLR } \\
\hline (1) Continuous & 1.156 & $1.037-1.289$ & 0.009 & & & \\
\hline (2) High versus low NLR & & & & 2.566 & $1.348-4.887$ & 0.004 \\
\hline
\end{tabular}

NLR, neutrophil-to-lymphocyte ratio; low NLR, <3.7; high NLR, $\geq 3.7$; BMI, body mass index; ECOG PS, Eastern Cooperative Oncology Group performance status; cRCC, clear cell renal cell carcinoma; pRCC, papillary renal cell carcinoma; chRCC, chromophobe renal cell carcinoma; HR, hazard ratio; CI, confidence interval.

multivariate models for RFS and CSS increased by $2.2 \%$ and $4.2 \%$, respectively, with NLR inclusion.

The present study had several strengths, compared to the previous studies in the field (Table 1). Firstly, this was the largest study that included the three major histologic subtypes of RCC. Secondly, while the present study evaluated both RFS and CSS, most of the previous studies did not evaluate CSS. The identification of CSS as well as RFS is a corner stone to prove the prognostic value of NLR. Finally, the present study included the most widely accepted independent prognostic factors of non-mRCC, including age, gender, and BMI; ECOG PS; symptoms at presentation; tumor size, stage, and grade; histologic subtype, sarcomatoid differentiation, and tumor necrosis.

In terms of clinical and pathologic characteristics at diagnosis, patients with high NLR differed significantly from those with low NLR in various parameters. Patients with high NLR had a larger tumor, a higher T stage, worse ECOG PS, worse symptoms, sarcomatoid differentiation, and tumor necrosis. These results are similar to those reported in previous studies $[17,18,20]$, suggesting that higher NLR may be associated with worse clinical behavior of non-mRCC.

NLR was shown to be a possible prognostic factor for mRCC in multiple studies, irrespective of the treatment method [8, 11-13]. However, studies concerning the prognostic significance of NLR for non-mRCC are scarce, with conflicting results. Some studies did not show a relationship between NLR and non-mRCC prognosis [16, 22], while others did [14, 15, 18-21]. Interestingly, one study reported different results for RFS and CSS [17]. These conflicting results may partly be because previous studies were relatively small-scale and lacked other possible prognostic factors as confounding variables (Table 1).

An important point is that most of the previous studies incorporated NLR as a categorical variable in their models. The use of a continuous variable reflects an intrinsic effect, whereas that of a categorical variable seems to adjust itself and to be created [23]. In addition, it is difficult to interpret the prognostic value of NLR using different cut-off levels, although most studies including the present one showed that the cut-off levels of NLR were in the range 3-4 (Table 1). In this respect, it is remarkable that NLR was not only used as a categorical variable but also as a continuous variable in this study. We identified that NLR as a continuous variable was also an independent prognostic factor. Interestingly, NLR cut-off level of 3.7 was estimated for CSS as well as RFS in this study. Considering that CSS is in alignment with RFS in non$\mathrm{mRCC}$, these results may strengthen our conclusion.

It is well known that inflammation affects tumorigenesis and progression $[3,17]$. Neutrophils represent the inflammatory response, whereas lymphocytes reflect cell-mediated immunity [3]. Therefore, a high NLR reflects both an increased inflammatory and a decreased antitumor immune response, suggesting a possible contribution to aggressive 
tumor biology, tumor progression, and poor survival [17]. In various cancers including hepatocellular carcinoma and colorectal cancer, high NLR was associated with poor outcome $[9,10]$. This was also supported by the results of our clinical study, which showed that higher NLR was likely to be associated with worse clinical behavior and indicated poor prognosis for RFS and CSS.

In contrast to our findings, some studies did not show a relationship between NLR and non-mRCC prognosis [16, 22]. In a study of 678 patients with cRCC, Pichler et al. [16] reported that NLR was not an independent prognostic factor for CSS or metastasis-free survival. However, NLR was only included as a categorical variable in this analysis. Certainly, a specified cut-off level may create a false or misleading association. Furthermore, they only analyzed patients with cRCC. As RCC is a heterogeneous and complex disease [24, 25], its results may not be directly applicable to patients with noncRCC. In a study of 228 patients with non-mRCC, Jagdev et al. [22] reported that NLR was not an independent prognostic factor for disease-free survival. However, their study involved only a small number of patients. Furthermore, as their study did not focus on NLR, the data on NLR were insufficient and were logarithmically transformed for analysis.

This study also had a few limitations. Firstly, data were retrospectively collected. Secondly, preoperative conditions such as chronic infection and chronic disease, which might affect the level of NLR, were not included. However, it is impossible to identify all the conditions associated with the NLR level in the clinical setting. Therefore, this study may be a better representation of the prognostic significance of NLR in actual practice. Lastly, this study lacked a central review of pathology, although most of the previous large multicenter studies did. Instead, urologic pathologists determined all pathologic features at each institution.

Despite limitations, it is noted that this study is the largest in the field, incorporating the most widely accepted independent prognostic factors of non-mRCC and evaluating both RFS and CSS.

\section{Conclusion}

This study showed that patients with high NLR differed significantly from those with low NLR in various clinical and pathologic parameters, suggesting that higher NLR may indicate worse clinical behavior of non-mRCC. In addition, NLR was a significant prognostic factor of both RFS and CSS, and incorporation of NLR into conventional prognostic predictors increased the predictive accuracy by $2.2 \%$ and $4.2 \%$, respectively. This study suggests that the use of preoperative NLR may be helpful in counseling and clinical trial design in patients with non-mRCC.

\section{Abbreviations}

Non-mRCC: Nonmetastatic renal cell carcinoma

NLR: $\quad$ Neutrophil-to-lymphocyte ratio

ANC: $\quad$ Absolute neutrophil count

ALC: $\quad$ Absolute lymphocyte count

ECOG PS: Eastern Cooperative Oncology Group performance status
TNM: Tumor-node-metastasis

WHO: World Health Organization

RFS: Recurrence-free survival

CSS: Cancer-specific survival

BMI: Body mass index

HR: Hazard ratio

CI: Confidence interval.

\section{Competing Interests}

The authors have nothing to disclose.

\section{Authors' Contributions}

Seok-Soo Byun participated in the study's design, coordination, treatment of patients, and data collection. Eu Chang Hwang, Seok Ho Kang, Sung-Hoo Hong, Jinsoo Chung, Tae Gyun Kwon, Hyeon Hoe Kim, Cheol Kwak, and Yong-June Kim were members of the research group and participated in the treatment of patients and data collection. Won Ki Lee conceived the study, participated in its design, performed the statistical analysis, and drafted the manuscript. All authors read and approved the final manuscript.

\section{References}

[1] R. L. Siegel, K. D. Miller, and A. Jemal, "Cancer statistics, 2015," CA Cancer Journal for Clinicians, vol. 65, no. 1, pp. 5-29, 2015.

[2] B. Ljungberg, K. Bensalah, S. Canfield et al., "EAU guidelines on renal cell carcinoma: 2014 update," European Urology, vol. 67, no. 5, pp. 913-924, 2016.

[3] S. I. Grivennikov, F. R. Greten, and M. Karin, "Immunity, Inflammation, and Cancer," Cell, vol. 140, no. 6, pp. 883-899, 2010.

[4] C. S. D. Roxburgh and D. C. McMillan, "Role of systemic inflammatory response in predicting survival in patients with primary operable cancer," Future Oncology, vol. 6, no. 1, pp. 149163,2010

[5] R. Zahorec, "Ratio of neutrophil to lymphocyte counts-rapid and simple parameter of systemic inflammation and stress in critically ill," Bratislavske Lekarske Listy, vol. 102, no. 1, pp. 5-14, 2001.

[6] P. H. Gibson, B. L. Croal, B. H. Cuthbertson et al., "Preoperative neutrophil-lymphocyte ratio and outcome from coronary artery bypass grafting," American Heart Journal, vol. 154, no. 5, pp. 995-1002, 2007.

[7] B. Azab, V. Chainani, N. Shah, and J. T. McGinn, "Neutrophillymphocyte ratio as a predictor of major adverse cardiac events among diabetic population: a 4-year follow-up study," Angio$\log y$, vol. 64, no. 6, pp. 456-465, 2013.

[8] K. Hu, L. Lou, J. Ye, and S. Zhang, "Prognostic role of the neutrophil-lymphocyte ratio in renal cell carcinoma: a metaanalysis," BMJ Open, vol. 5, no. 4, Article ID e006404, 2015.

[9] M.-X. Li, X.-M. Liu, X.-F. Zhang et al., "Prognostic role of neutrophil-to-lymphocyte ratio in colorectal cancer: a systematic review and meta-analysis," International Journal of Cancer, vol. 134, no. 10, pp. 2403-2413, 2014.

[10] W.-K. Xiao, D. Chen, S.-Q. Li, S.-J. Fu, B.-G. Peng, and L.-J. Liang, "Prognostic significance of neutrophil-lymphocyte ratio in hepatocellular carcinoma: a meta-analysis," BMC Cancer, vol. 14, no. 1, article 117, pp. 117-126, 2014. 
[11] J. Atzpodien, P. Royston, T. Wandert, and M. Reitz, "Metastatic renal carcinoma comprehensive prognostic system," British Journal of Cancer, vol. 88, no. 3, pp. 348-353, 2003.

[12] F. Donskov and H. von der Maase, "Impact of immune parameters on long-term survival in metastatic renal cell carcinoma," Journal of Clinical Oncology, vol. 24, no. 13, pp. 1997-2005, 2006.

[13] D. Keizman, M. Ish-Shalom, P. Huang et al., “The association of pre-treatment neutrophil to lymphocyte ratio with response rate, progression free survival and overall survival of patients treated with sunitinib for metastatic renal cell carcinoma," European Journal of Cancer, vol. 48, no. 2, pp. 202-208, 2012.

[14] Y. Ohno, J. Nakashima, M. Ohori, T. Gondo, T. Hatano, and M. Tachibana, "Followup of neutrophil-to-lymphocyte ratio and recurrence of clear cell renal cell carcinoma," Journal of Urology, vol. 187, no. 2, pp. 411-417, 2012.

[15] I. Lucca, M. de Martino, S. L. Hofbauer, N. Zamani, S. F. Shariat, and T. Klatte, "Comparison of the prognostic value of pretreatment measurements of systemic inflammatory response in patients undergoing curative resection of clear cell renal cell carcinoma," World Journal of Urology, vol. 33, no. 12, pp. 2045-2052, 2015.

[16] M. Pichler, G. C. Hutterer, C. Stoeckigt et al., "Validation of the pre-treatment neutrophil-lymphocyte ratio as a prognostic factor in a large European cohort of renal cell carcinoma patients," British Journal of Cancer, vol. 108, no. 4, pp. 901-907, 2013.

[17] B. R. Viers, R. H. Thompson, S. A. Boorjian, C. M. Lohse, B. C. Leibovich, and M. K. Tollefson, "Preoperative neutrophil-lymphocyte ratio predicts death among patients with localized clear cell renal carcinoma undergoing nephrectomy," Urologic Oncology: Seminars and Original Investigations, vol. 32, no. 8, pp. 1277-1284, 2014.

[18] J. Huang, D. M. Dahl, L. Dong et al., "Preoperative neutrophilto-lymphocyte ratio and neutrophilia are independent predictors of recurrence in patients with localized papillary renal cell carcinoma," BioMed Research International, vol. 2015, Article ID 891045, 9 pages, 2015.

[19] M. De Martino, A. J. Pantuck, S. Hofbauer et al., "Prognostic impact of preoperative neutrophil-to-lymphocyte ratio in localized nonclear cell renal cell carcinoma," Journal of Urology, vol. 190, no. 6, pp. 1999-2004, 2013.

[20] R.-M. Wen, Y.-J. Zhang, S. Ma et al., "Preoperative neutrophil to lymphocyte ratio as a prognostic factor in patients with nonmetastatic renal cell carcinoma," Asian Pacific Journal of Cancer Prevention, vol. 16, no. 9, pp. 3703-3708, 2015.

[21] P. Forget, J.-P. Machiels, P. G. Coulie et al., "Neutrophil: lymphocyte ratio and intraoperative use of ketorolac or diclofenac are prognostic factors in different cohorts of patients undergoing breast, lung, and kidney cancer surgery," Annals of Surgical Oncology, vol. 20, no. 3, supplement, pp. S650-S660, 2013.

[22] S. P. K. Jagdev, W. Gregory, N. S. Vasudev et al., "Improving the accuracy of pre-operative survival prediction in renal cell carcinoma with C-reactive protein," British Journal of Cancer, vol. 103, no. 11, pp. 1649-1656, 2010.

[23] Y. Luo, D.-L. She, H. Xiong, S.-J. Fu, and L. Yang, "Pretreatment neutrophil to lymphocyte ratio as a prognostic predictor of urologic tumors: a systematic review and meta-analysis," Medicine, vol. 94, no. 40, article e1670, 2015.

[24] S.-S. Byun, S. K. Hong, S. Lee et al., "The establishment of KORCC (Korean Renal Cell Carcinoma) database," Investigative and Clinical Urology, vol. 57, no. 1, pp. 50-57, 2016.
[25] F.-M. Deng and J. Melamed, "Histologic variants of renal cell carcinoma: does tumor type influence outcome?" Urologic Clinics of North America, vol. 39, no. 2, pp. 119-132, 2012. 


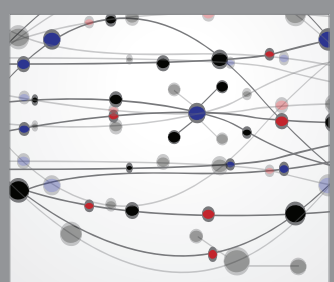

The Scientific World Journal
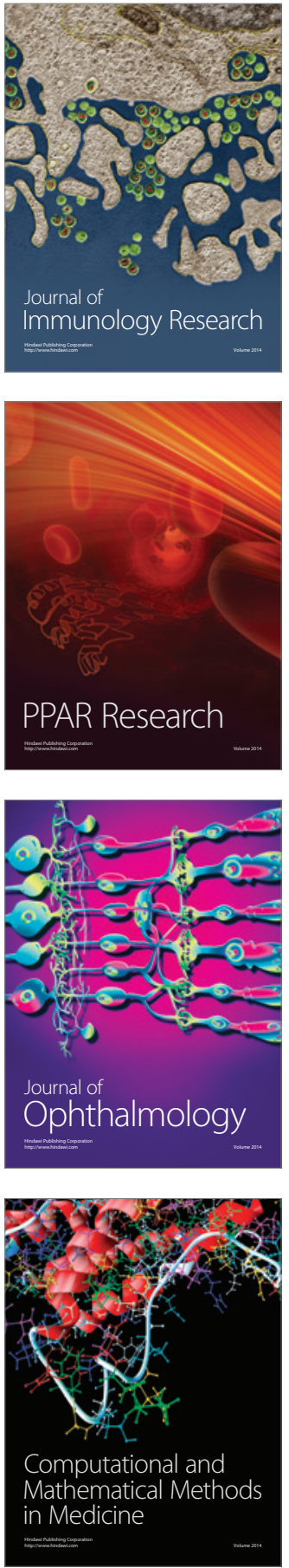

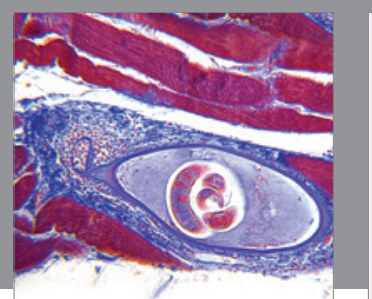

Gastroenterology Research and Practice

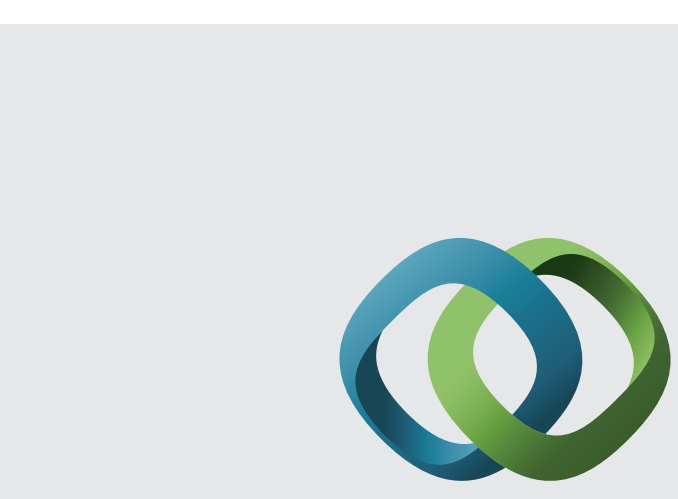

\section{Hindawi}

Submit your manuscripts at

http://www.hindawi.com
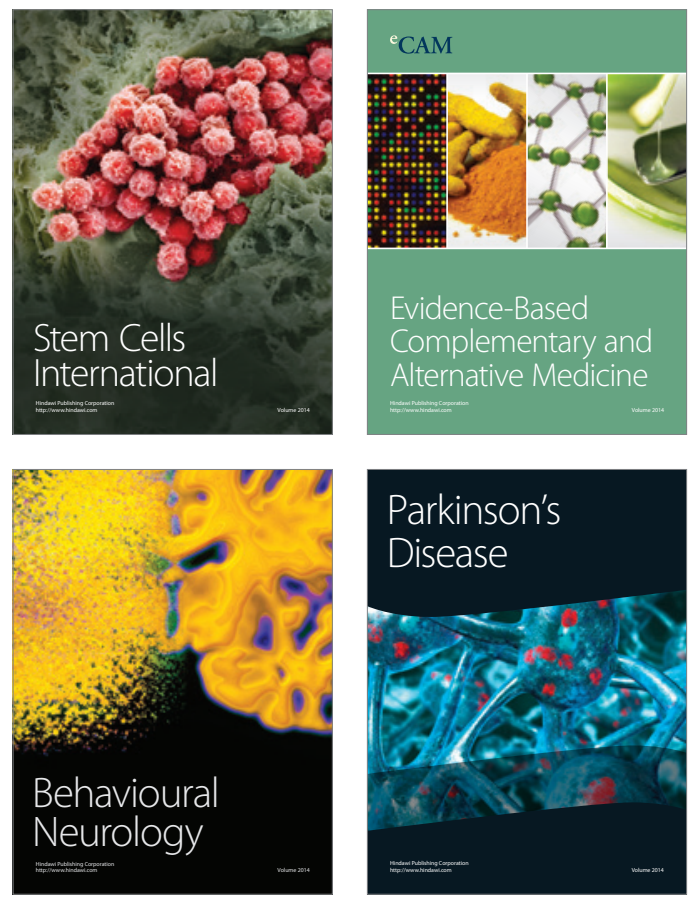
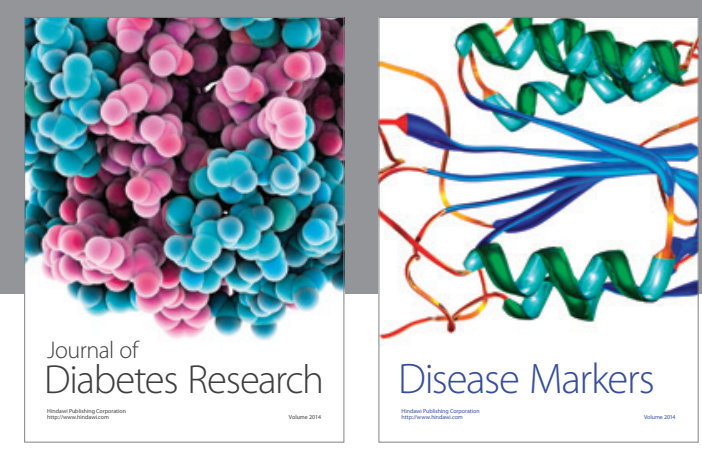

Disease Markers
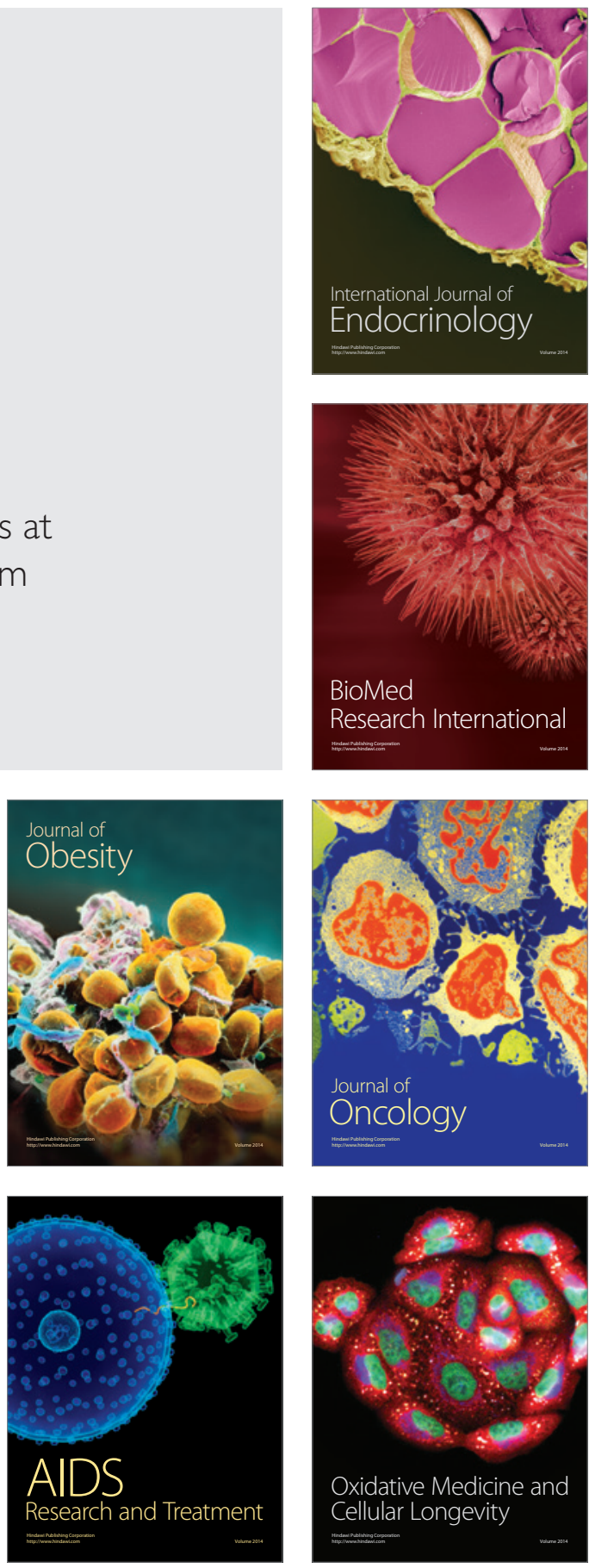